\title{
Design and Manufacture of Child Prosthesis for Higher Members Below the Elbow Through Reverse Engineering
}

\section{Diseño y Manufactura de Prótesis Infantiles para Miembros Superiores por Debajo del Codo Mediante Ingeniería Inversa}

\author{
E. Pozo ${ }^{1}$, E. Medina ${ }^{2}$, and A. Pazmiño ${ }^{1}$
}

VII International Congress of Science, Technology, Entrepreneurship and Innovation (SECTEI 2020)

Corresponding Author:

E. Pozo

edwin.pozo@espoch.edu.ec

Published: 26 August 2021

Production and Hosting by

Knowledge E

(c) E. Pozo et al. This article is distributed under the terms of the Creative Commons

Attribution License, which permits unrestricted use and redistribution provided that the original author and source are credited.
${ }^{1}$ Docente de la Facultad de Mecánica, Escuela Superior Politécnica de Chimborazo, Riobamba, Ecuador

${ }^{2}$ Técnico de Investigación. Instituto de Investigación, Escuela Superior Politécnica de Chimborazo, Ecuador

\section{Abstract}

The objective was to build a prosthesis with its elements such as socket, forearm and hand. The prostheses that are below the elbow generated the motivation for the investigation, being an important factor the correct adaptation of the socket to the stump in a personalized way creating an adjustment due to the vacuum that is generated between these elements, without the need to use a harness. For the design and construction of the prosthesis prototype, a Kinect device was used to obtain a point cloud of the geometric model, the alginate for the manufacture of the mold and plaster that is used to obtain the shape of the hand. The result is an individualized prosthesis design built based on the patient's own dimensions and validated using finite elements; with the purpose of making changes and improvements in the design, to have a prosthesis of easy placement and use.

Keywords: prosthesis, socket, stump, finite elements, kinect.

\section{Resumen}

El objetivo fue construir una prótesis con sus elementos como son socket, antebrazo y la mano. Las prótesis que estén por debajo del codo genero la motivación de la investigación, siendo un factor importante la correcta adaptación del socket al muñón de forma personalizada creando un ajuste debido al vacío que se genera entre dichos elementos, sin la necesidad de utilizar un arnés. Para el diseño y construcción del prototipo de prótesis, se utilizó un dispositivo Kinect para la obtención de una nube de puntos del modelo geométrico, el alginato para la fabricación del molde y yeso que es utilizado para la obtención de la forma de la mano. Se presenta como resultado un diseño individualizado de prótesis construida en base a las dimensiones propias del paciente y validado mediante elementos finitos; con el propósito de realizar cambios y mejoras en el diseño, para tener una prótesis de fácil colocación y uso.

Palabras Clave: prótesis, socket, muñón, elementos finitos, kinect. 


\section{Introducción}

En la vida diaria si a una persona le falta una parte del cuerpo para poder desenvolverse de manera normal, se recomienda el uso de un dispositivo artificial (prótesis) para reemplazarla, generando la oportunidad de continuar con una vida normal. Una prótesis debe permitir a la persona realizar, como mínimo, las actividades cotidianas (como caminar, comer y vestirse) de forma independiente y cómoda. Entonces una prótesis permite al usuario funcionar tan bien como el miembro no amputado [1].

Los miembros superiores son una excelente herramienta para la persona, se componen del hombro, brazo, codo, antebrazo y mano que en conjunto son capaces de ejecutar innumerables acciones gracias a sus movimientos, dentro de sus principales funciones es mantener la estabilidad y las actividades de sujeción.

La prótesis está dotada de una gran riqueza funcional que procura una abundancia de posibilidades en las posiciones, los movimientos y las acciones que simulan al miembro no amputado [2].

Los tipos de prótesis varían de acuerdo al nivel de amputación, la función que va ejercer el material y el método de fabricación. Tomando en cuenta cada nivel de amputación las prótesis pueden ser (para muñeca, codo u hombro) o debajo o arriba de la articulación, según la función a ejercer, las prótesis se dividen en dos tipos: Miembros artificiales pasivos o cosméticos y miembros activos o funcionales [3].

Las prótesis pasivas son creadas principalmente para reestablecer la imagen corporal del paciente. La principal y única función de este tipo de prótesis es satisfacer los deseos del paciente respecto al peso, apariencia física, material, conformación y comodidad de la prótesis. Tienen movimientos simples y funcionales y pueden ser:

1. Cosmética de dedo: Su función es remplazar la ausencia de uno varios dedos.

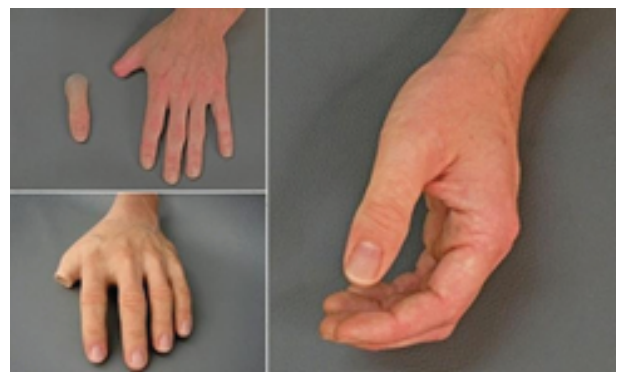

\section{Figure 1}

Prótesis cosmética de dedo. Fuente:

https://www.dailymail.co.uk/health/article-2694703/The-fingers-thumbs-youd-never-know-pro sthetics-German-designers-create-remarkably-life-like-body-parts-silicone.html

(2) Cosmética de manos o muñeca: Este tipo de prótesis se ocupa cuando hay ausencia parcial o total de la mano. El diseño de estas prótesis es que los dedos o 
manos deben ser lo más parecido a la forma, color y textura de la piel del individuo todo este se logra con el diseño por computadora [4].

(3) Cosmética de manos o muñeca: Este tipo de prótesis se ocupa cuando hay ausencia parcial o total de la mano. El diseño de estas prótesis es que los dedos o manos deben ser lo más parecido a la forma, color y textura de la piel del individuo todo este se logra con el diseño por computadora.
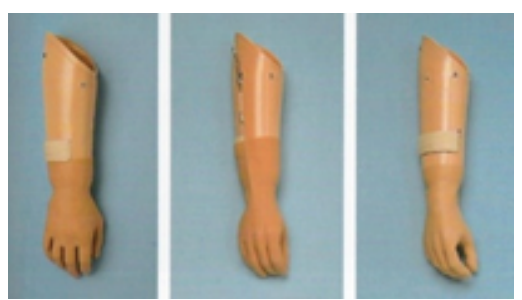

Figure 2

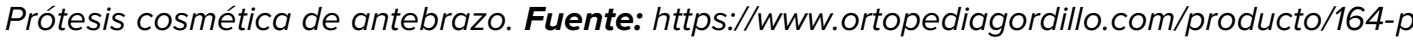
rotesis-amputacion-de-brazo-estetico-pasiva-encaje-laminado-o-similar-sistema-endoesque letico-funda-estetica-y-correaje-de-suspension-mano-estetica-pasiva-y-guante-cosmetico/

(4) Cosmética de brazo: Se utiliza en cualquier nivel en el brazo. En el diseño se debe crear cavidad conforme a las mediciones del muñón, todos los compontes se adaptan, se perfecciona la forma estética mediante el diseño CAD.

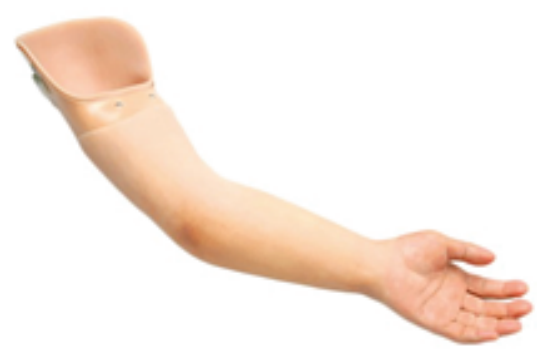

Figure 3

Prótesis cosmética de brazo. Fuente:

https://www.ortopedialopez.com/tienda/estetico/mano-estetica-silicon-house/

(5) Cosmética de codo: Se ocupa cuando hay una amputación a nivel del codo. Su diseño es el mismo al indicado en la 'Cosmética de brazo'.

(6) Cosmética para desarticulado de hombro: Esta prótesis se usa cuando existen amputaciones de desarticulación humeral y exarticulación clavícula-escapular. Su diseño es el mismo al indicado en la 'Cosmética de brazo' [5]. 


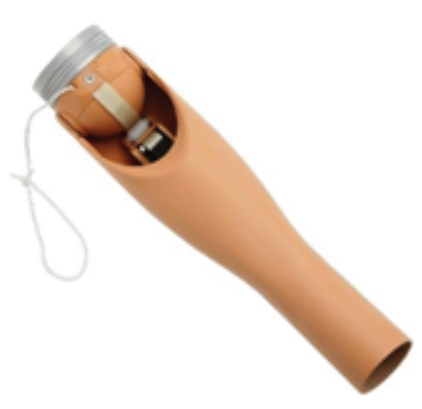

\section{Figure 4}

Prótesis cosmética de codo. Fuente:

https://www.ottobock.es/protesica/miembro-superior/sistemas-de-brazo-y-mano/ergoarm/

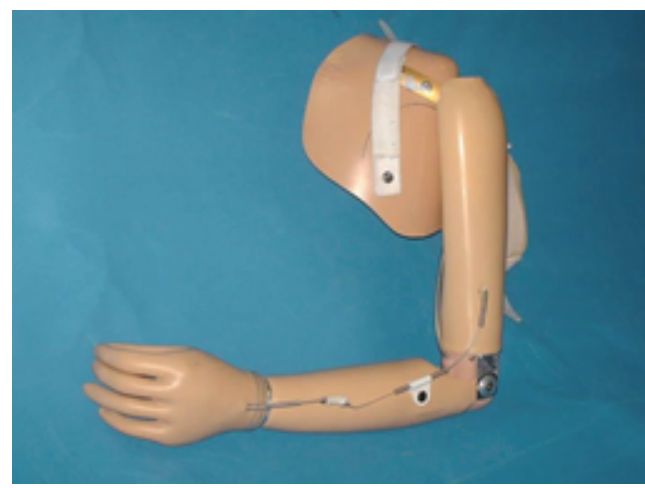

\section{Figure 5}

Prótesis cosmética para desarticulado de hombro. Fuente: https://www.protexintegral.cl/protesis-cakk

Las prótesis activas son aquellas que llegan a sustituir al miembro amputado de forma funcional. Se pueden dividir en tres: de fuerza propia, de fuerza ajena e híbridas. En las prótesis de fuerza propia se utiliza los movimientos del cuerpo, y para realizar las funciones mecánicas se utiliza un sistema de cables y otros componentes mecánicos [6].

Los miembros artificiales de fuerza ajena son los que utilizan motores y electrodos que ayudan a captar y transformar los voltajes que reciben en movimientos. Por último, las híbridas son una combinación de las dos anteriores

\section{Materiales y Métodos}




\subsubsection{Polímero ABS}

El Acrilonitrilo Butadieno Estireno (ABS) es uno de los termoplástico más comunes. Cada uno de estos componentes le brinda una propiedad definida: El Acrilonitrilo da estabilidad ante el calor y las reacciones químicas y rigidez; el Butadieno proporciona protección contra los impactos y ofrece tenacidad ante las bajas temperaturas; el Estireno está presente para dar resistencia mecánica y brillo.

El ABS se derrite a $220^{\circ}$, pero puede comenzar a ser manipulable desde los $100^{\circ}$, y al enfriarse se transforma en un material duro, brillante y resistente contra los impactos. Está hecho a base de petróleo crudo y no es tóxico. Se puede pintar, reteniendo el color fácilmente, lijar, pulir, agujerar o pasar por diversos procesos industriales. Este material puede ser manipulado con gran facilidad, sin embargo, es muy difícil de romper. Una desventaja que se presenta es la liberación de químicos, tal vez tóxicos, cuando el ABS se calienta o se quema, además de no ser biodegradable, solo reciclable.

Cualquier producto fabricado con ABS será duro y resistente al agua y a los químicos, sin embargo, no se recomienda para uso al aire libre. El costo de este material oscila entre 20 y 50 dólares por kilogramo [7].

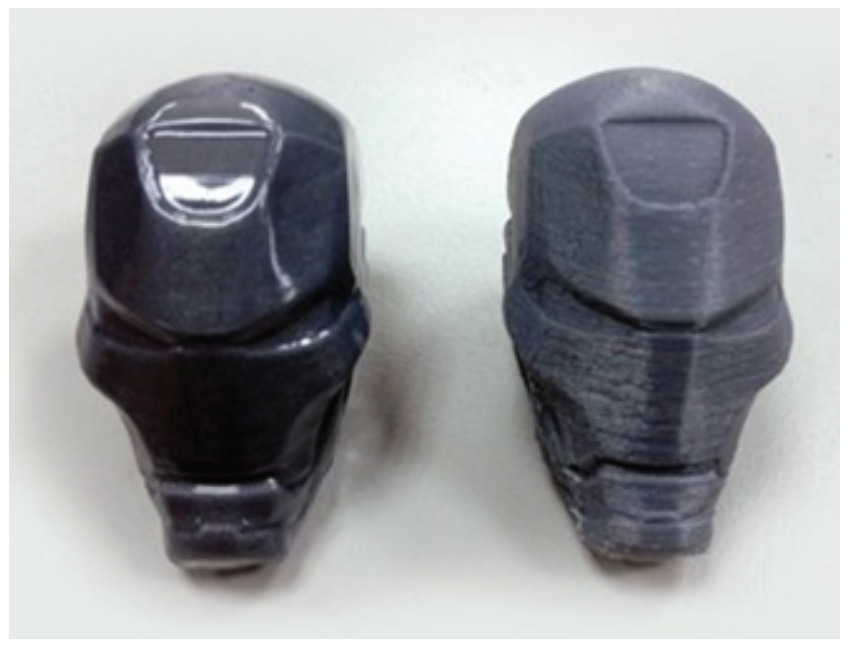

\section{Figure 6}

Material de impresión 3D ABS. Fuente: https:

//www.kickstarter.com/projects/magicbox/magicbox-magically-finish-your-3d-prints?lang=es

\subsubsection{Polímero PLA}

El Ácido Poli láctico (PLA) es un polímero relativamente nuevo, está hecho a base de materiales biológicos como almidón o caña de azúcar. El material es parecido al que se utiliza en los plásticos de empaque biodegradables y se derrite a una temperatura entre $180^{\circ}$ y $200^{\circ}$, dependiendo del material que se agregue para color y textura. Una 
vez frío, el PLA endurece y forma un material resiliente mate opaco. Su tolerancia al calor no es mayor que la del ABS, ya que su deformación puede comenzar desde los $60^{\circ}$. A diferencia del anterior, el PLA no tiene un aroma tan fuerte cuando se expone a altas temperaturas [7].

EI PLA es la opción más común para impresoras 3D económicas; se imprime con mayor facilidad que con el ABS porque se pega mejor a otras superficies y con él mismo. Otra ventaja es la biodegradabilidad que se presenta cuando el material se encuentra bajo la superficie terrestre, aunque su tiempo de vida sea prolongado en condiciones normales. No es seguro usar las impresiones de PLA para que estén en contacto con la comida y no tiene tanta resistencia al calor como el ABS. El PLA es más frágil, lo que significa que, bajo la aplicación de estrés, los productos se pueden fisurar o romper; sin embargo, se pueden agregar químicos para hacer más resistente al PLA (se le llama PLA duro). Su costo es igual al del ABS (\$20 a 50 por kg).

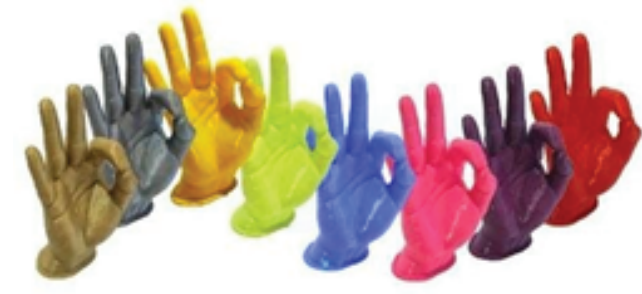

Figure 7

Material de impresión 3D PLA. Fuente:

https://www.milanuncios.com/impresoras/filamentos-pla-gold-1-75mm-1-kg-195212950.htm

\subsubsection{El alcohol polivinilo PVA}

El Alcohol Polivinilo (PVA) es un tipo de material de impresión nuevo y sirve, más que nada, para fabricar soportes. Se trata de un polímero sintético utilizado en productos biodegradables que tienen una función, pero que al terminarla se deben disolver. El PVA se derrite a $200^{\circ}$ y si se sobrecalienta puede liberar químicos tóxicos; además, es soluble en agua. Esta última propiedad es el motivo por el cual se utiliza para imprimir soportes. Una vez que el modelado complejo esté terminado, se sumerge en agua para disolver los soportes que cumplieron su función en la construcción del producto. La propiedad de disolución le atribuye al PVA la característica de ser costoso (pueden ser más de cien dólares por kg).

\subsubsection{Filamento flexible}

El material para impresiones en 3D, generalmente, busca la rigidez y fortaleza de un sólido. Sin embargo, hay ocasiones en donde no es eso lo que se necesita. Para estos 


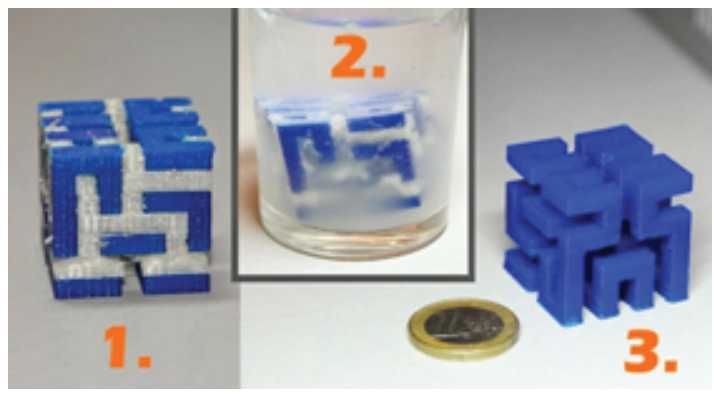

Figure 8

Material de impresión 3D PVA. Fuente: http:

//www.3dprintingstore.co.za/filament/cron/cron-speciality/cron-pva-filament-0-5kg-1-75mm/

casos existen materiales como el Ninjaflex, el Polyflex o el Trueflex que fabrican impresiones flexibles con la capacidad de estirarse. Este tipo de material tiene aplicaciones biomédicas como articulaciones en las prótesis o como partes del cuerpo que tienen que reacomodarse según la posición en la que se encuentre. La desventaja al usar filamentos flexibles es la complejidad a la hora de imprimir porque cuando el material tiene esta característica es más difícil ser extruido y la impresora se tiene que modificar para facilitar el proceso. Al igual que los filamentos de fibra de carbono, su costo oscila entre y $\$ 50$ y 120 por $\mathrm{kg}[8]$.

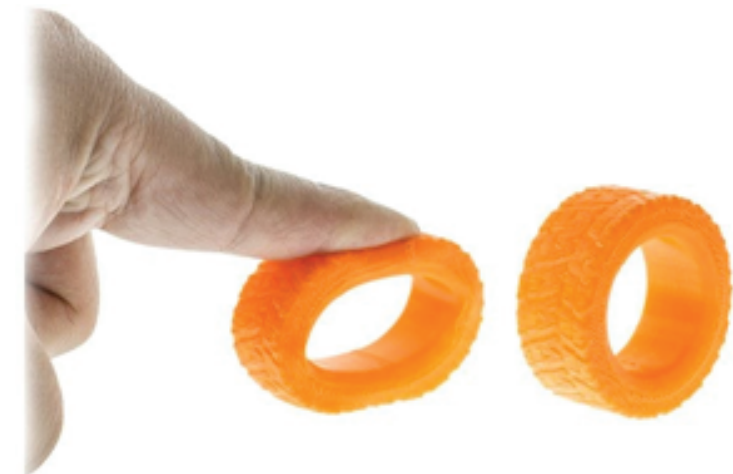

Figure 9

Material de impresión 3D FLEXIBLE. Fuente:

https://dressmodelanarkali.blogspot.com/2019/05/unix-polyflex-printable-sablon-punk.html

\subsection{Escaneo del brazo para el diseño del muñón}

Para digitalización del brazo se utiliza el Kinect Xbox 360 como escáner 3D, una vez se conecta el Kinect a la computadora, se configura los parámetros de escaneo en el software, como la distancia a la que debe estar el objeto del escáner que para este caso se estableció en 600 mm, la calidad de la malla tomamos media y los FPS en número de 25 para las capturas de las imágenes y de esa forma digitalizar el brazo en 
3D como se observa en la Figura 10. Se exporta el archivo el archivo OBJ al software Autodesk Recap Photo [9].

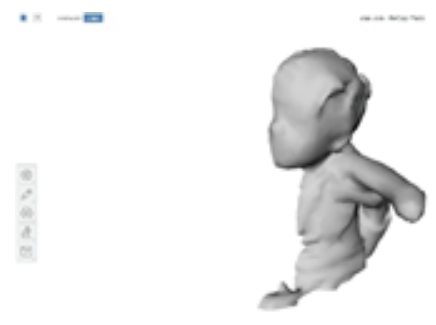

Figure 10

Escaneo completo. Fuente: Autores.

Una vez simplificadas las superficies escaneadas, se obtiene el área donde se concentra la edición del modelo en 3D, exportamos la malla como OBJ (QUADS) para transformar la malla triangular a malla cuadrada, es necesario cambiar el elemento de triángulos a cuadriláteros el software CAD fusión 360 reconoce solo elementos de malla cuadrada para poder convertir luego la malla en un sólido como se muestra en las Figuras 11,12 y 13. [10].

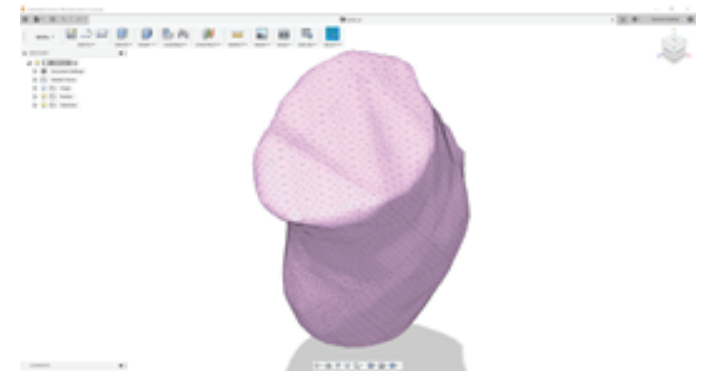

Figure 11

Archivo stl malla triangular. Fuente: Autores.

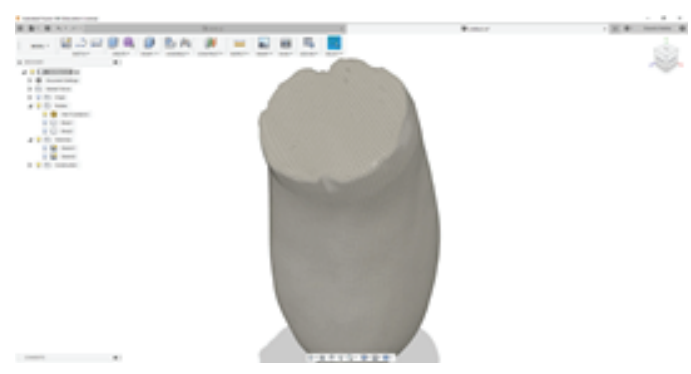

Figure 12

Malla cuadrada. Fuente: Autores.

El proceso de diseño del muñón se lo realiza sobre la superficie del solido obtenido de la malla cuadrada, el muñón toma la forma del miembro del brazo amputado, de 


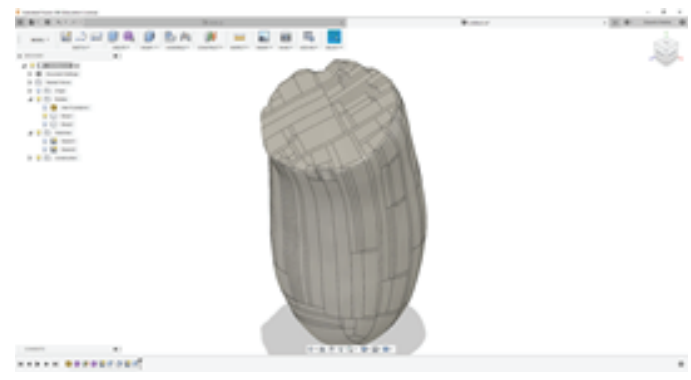

Figure 13

Archivo CAD elemento sólido. Fuente: Autores.

esa forma se efectúa un vacío entre el miembro amputado y el muñón, sin necesidad de correas o arnés para sujetar la prótesis [11].

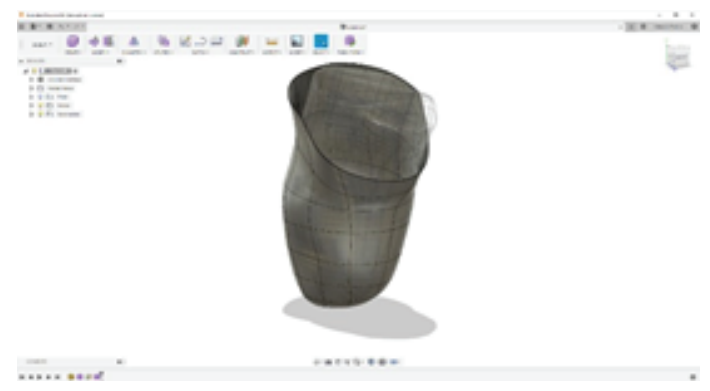

Figure 14

Archivo CAD elemento superficie, Muñón. Fuente: Autores.

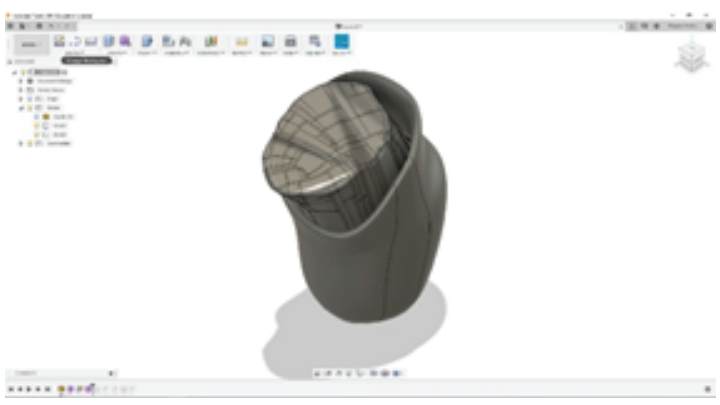

Figure 15

Archivo CAD elemento sólido, Muñón. Fuente: Autores.

\subsection{Impresión 3D del muñón}

Para obtener el objeto físico del diseño del muñón se exporta la geometría con la extensión. STL, este es el formato de lectura del software de impresión 3D en material PLA [12].

Las configuraciones para su impresión en 3D son: 


\section{Table 1}

Configuración de la impresora 3D.

Altura de la capa
Cantidad de relleno
Grosor de pared
Temperatura del extrusor
Temperatura de la cama
Velocidad de impresión

\section{$0,2 \mathrm{~mm}$}

$30 \%$

0,8

$230^{\circ} \mathrm{C}$

$60^{\circ} \mathrm{C}$

$60 \%$

Fuente: Autores.

Una vez se configura la pieza a imprimir se lo exporta con la extensión. GCODE pues este código genera coordenadas que la impresora lee para así aportar material e ir formando la pieza.

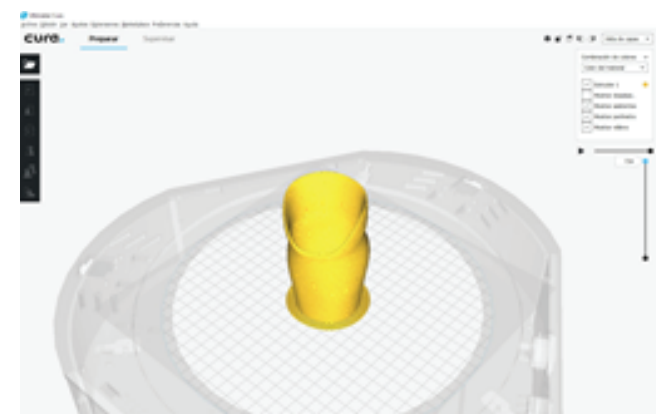

Figure 16

Archivo GCODE. Fuente: Autores.

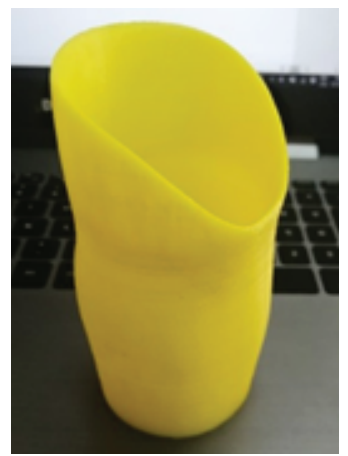

Figure 17

Archivo físico impreso en 3D. Fuente: Autores.

2.4. Diseño y fabricación de la mano 


\subsubsection{Elaboración de un molde yeso}

Se procede la elaboración de un molde en yeso de la mano derecha del paciente, para la reconstrucción de la mano faltante. Para esto se necesita dos materiales antes mencionado como son el alginato y el yeso.

Vertemos el polvo de alginato sobre el agua y seguido mezclamos muy bien usando una espátula. Es importante mezclar uno o dos minutos muy bien y de manera rápida antes de que empiece a catalizar la mezcla. Si se empieza a gelificar antes de su vertido podría estropearse el resultado. El líquido adquiere una consistencia viscosa para colocar en el molde preparado con anterioridad.

Una vez hecha la mezcla el paciente debe introducir la mano en la mezcla preparada para formar así el molde de la mano, se mezcla el yeso en recipiente aparte mezcla durante dos minutos cuando ya se tiene una mezcla homogénea se vierte el yeso en el molde de alginato y se deja por $12 \mathrm{hr}$ hasta que el yeso se endurece.

Se retira el alginato y queda la forma de la mano que es lo que se desea para realizar el diseño.

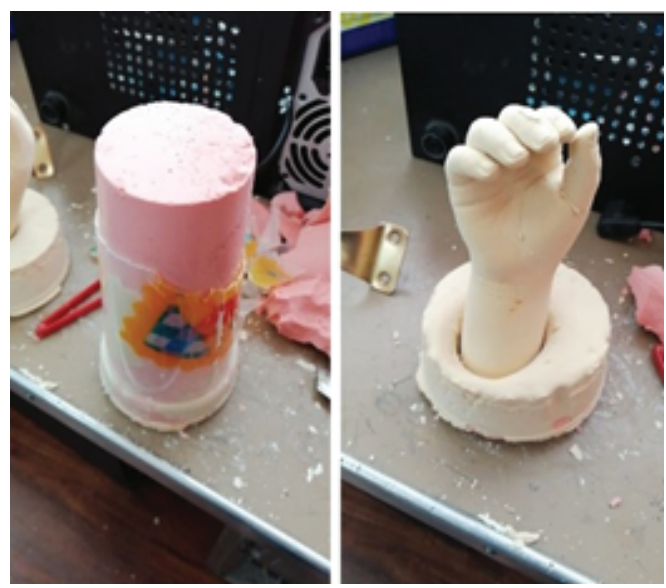

\section{Figure 18}

Molde de yeso. Fuente: Autores.

Una vez que se obtiene la forma de la mano, se procede al escaneo de la misma, para su respectivo diseño. Al escanear la mano solo se obtiene su contorno por la resolución del escáner es baja, para esto hay que corregir el archivo de forma manual y dar los detalles de la mano con la ayuda del software de dibujo CAD. Esta experimentación se realiza para evitar que en el momento de escanear genere problemas por falta de continuidad en el proceso y más aún cuando los pacientes son niños que por su condición generan movimientos involuntarios.

Con el modelo de la mano derecha se realiza una simetría a la geometría para obtener la mano izquierda y proceder a completar el brazo para la impresión de todas las partes de la prótesis. 


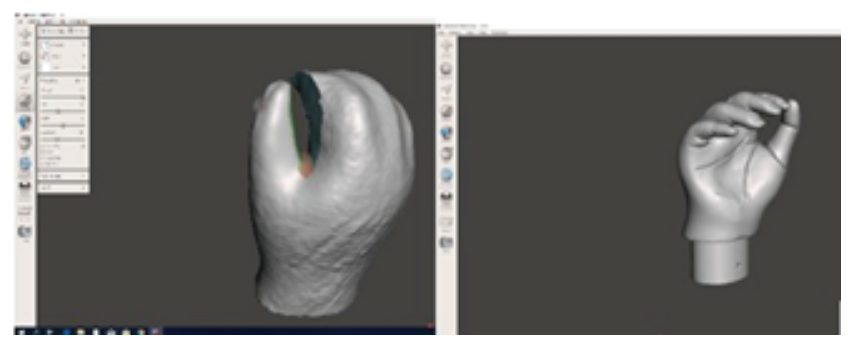

\section{Figure 19}

Diseño de la mano derecha. Fuente: Autores.
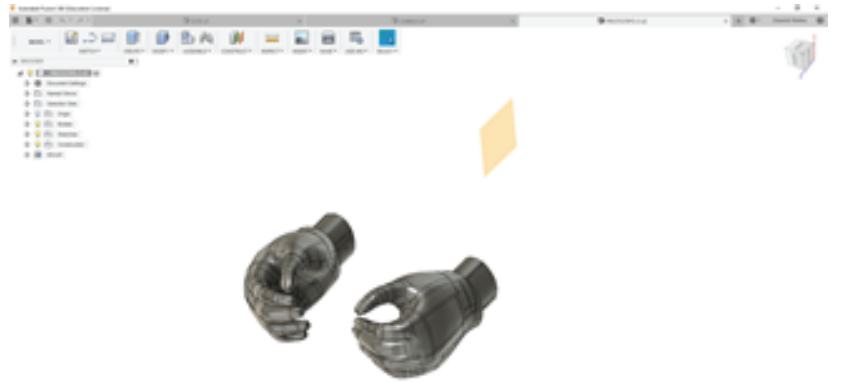

Figure 20

Obtención de la mano izquierda. Fuente: Autores.

Se realiza el modelado completo de la prótesis, y se imprime en 3D con filamento PLA.

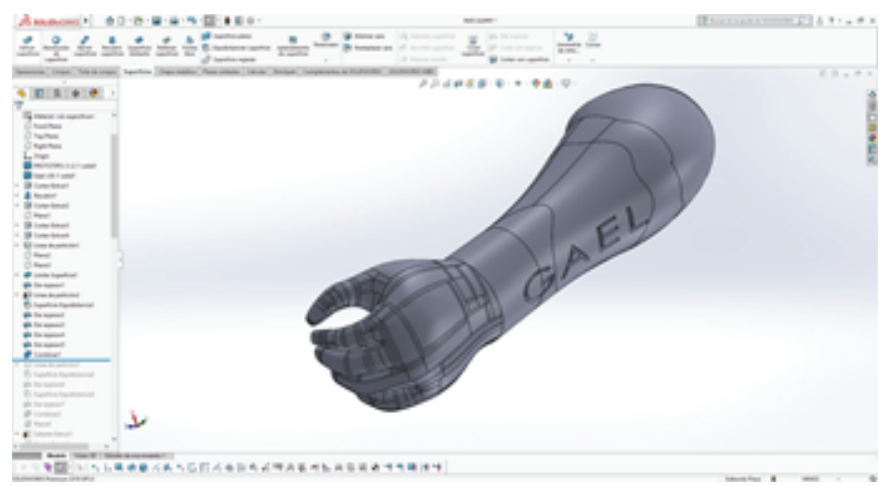

Figure 21

Diseño de la prótesis completa. Fuente: Autores.

\subsection{Impresión 3D de la mano y el brazo}

Para obtener el objeto físico del diseño de la prótesis completa se exporta la geometría con la extensión. STL, este es el formato de lectura del software de impresión 3D en material PLA [12]. 
Las configuraciones para su impresión en 3D se utiliza los parámetros de la Tabla 1.

Una vez se configura la pieza a imprimir se lo exporta con la extensión. GCODE pues este código proporciona coordenadas que la impresora lee para así aportar material e ir formando la pieza.

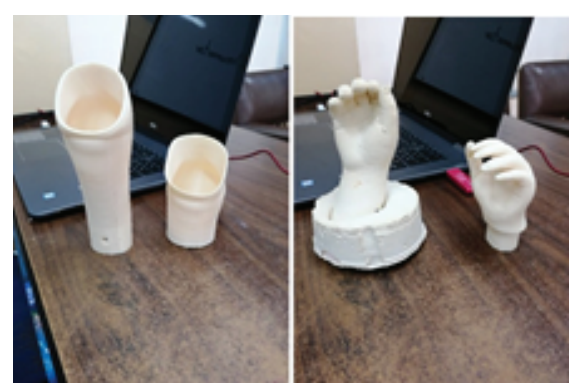

Figure 22

Impresión en 3D de la mano y el soque. Fuente: Autores.

La mano y el brazo están unidas mediante un tornillo quedando así acoplada.

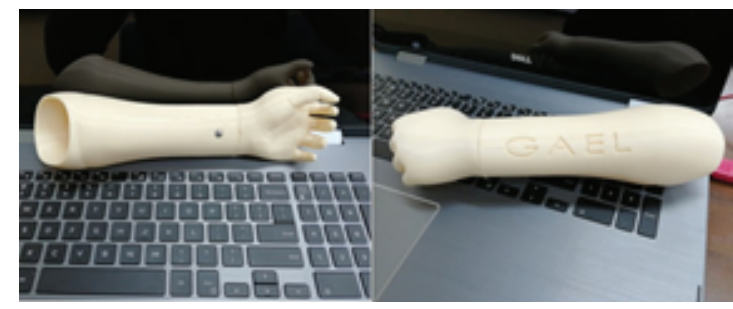

Figure 23

Prótesis completa. Fuente: Autores.

\subsection{Validación a través de simulación computacional}

\subsubsection{Definición del problema}

El modelo para simulación de la prótesis se obtuvo por medio la aplicación de herramientas de ingeniería inversa, por lo tanto, es importante tener la geometría totalmente definida evitando tener superficies o solidos entrecruzados, en la Figura 24 se muestra la prótesis para el análisis, el mismo que contendrá el material adecuado, restricciones y condiciones de carga a las que está sometido para obtener valores de deformación y esfuerzos [13].

\subsubsection{Pre proceso}




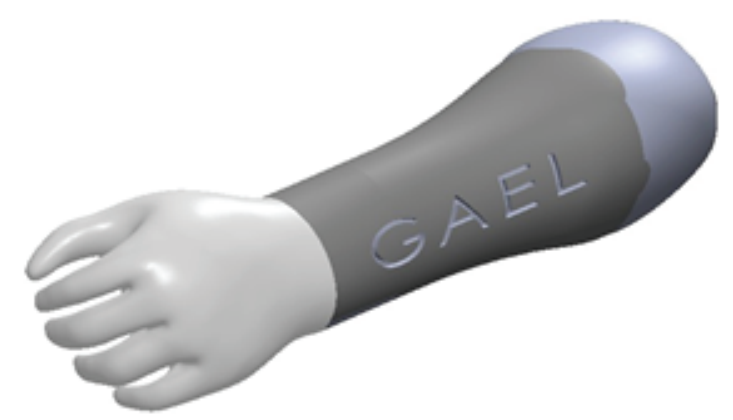

Figure 24

Prótesis para el análisis. Fuente: Autores.

\section{Definición de material}

La Figura 25 muestra las propiedades mecánicas del PLA, con las cuales se realiza el análisis computacional [14].

\begin{tabular}{|c|c|c|}
\hline Property & Volve & Unit \\
\hline [7. Material Feld & प Table & \\
\hline 8 Densty & 1.24 & $\operatorname{kg}=\wedge \cdot 3$ \\
\hline \multicolumn{3}{|l|}{ Z tsotropic Elastoty } \\
\hline Derive from & Young's Modulus an.... \pm & \\
\hline Young's Modulus & 3. $\mathbf{E}+09$ & Pa \\
\hline Poisson's Rato & 0.39 & \\
\hline Buk Modulus & $5.30 x+09$ & Po \\
\hline Shear Modulus & $1.259 \varepsilon+09$ & Po \\
\hline [A Tensle Yield Strength & 50 & Po \\
\hline 8 Compressive Yield & $\infty$ & Pa \\
\hline $\begin{array}{l}\text { Tensle Utimate } \\
\text { Strength }\end{array}$ & 60 & Pa \\
\hline
\end{tabular}

Figure 25

Propiedades mecánicas del PLA. Fuente: Autores.

\section{Definición de la malla}

Se genera la discretización para conseguir un número finito de pequeños tetraedros de control. Con la finalidad de observar la influencia de la discretización se generan varios modelos con distintos tipos de elementos entre ellos hexaedros. La opción de tomar los tetraedros es que se adapta al dominio, es decir a la forma [15].

\section{Definición del modelo físico}

Los diagramas de cuerpo libre se construyen para ayudar a identificar las fuerzas y los momentos que actúan sobre partes individuales de un sistema y para garantizar el uso 


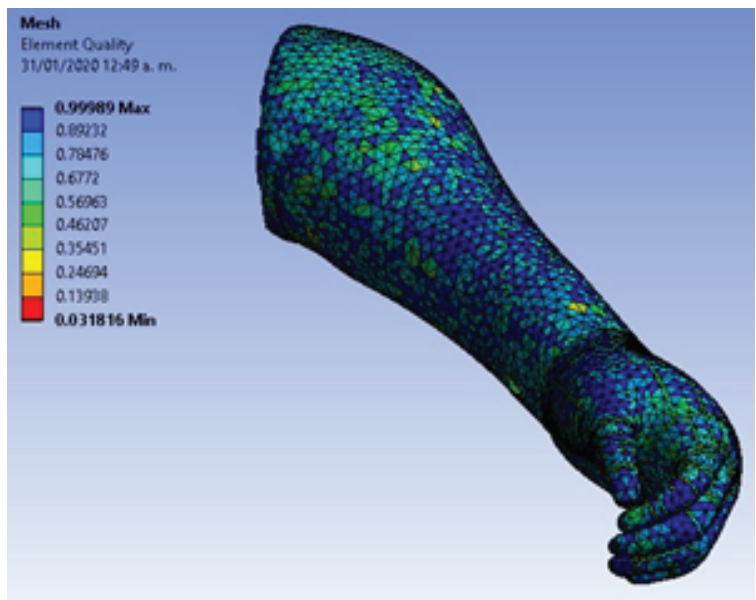

Figure 26

Malla con elementos tetraédricos. Fuente: Autores.

correcto de las ecuaciones de la mecánica para analizar el sistema. Para este propósito, las partes que constituyen un sistema están aisladas de su entorno y los efectos del entorno son reemplazados por fuerzas y momentos apropiados [16].

En algunos análisis, el objetivo puede ser investigar las fuerzas involucradas en y alrededor de varias articulaciones del cuerpo humano para diferentes condiciones posturales y de carga.

Dichos análisis pueden realizarse separando el cuerpo en dos partes en la articulación de interés y dibujando el diagrama de cuerpo libre de una de las partes.

Considerar a una persona en un aparato de ejercicio que sostiene un asa que está conectada a un cable como se muestra en la Figura 27.

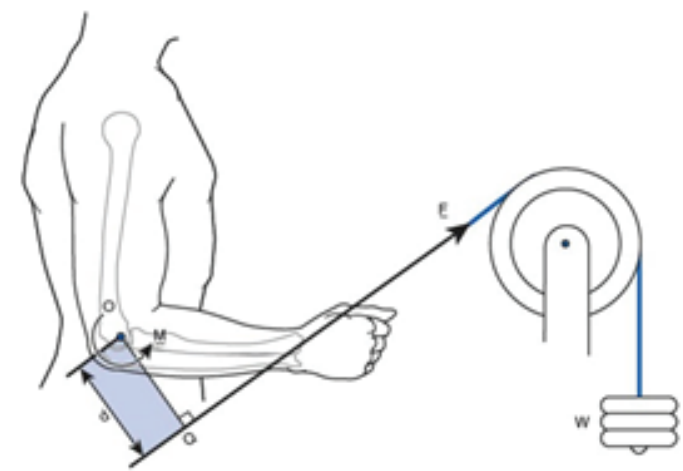

Figure 27

Definición de cargas. Fuente: Basic biomechanics of the musculoskeletal system.

$F$ es la fuerza aplicada a la mano por el asa del cable unido al peso en el plato de pesaje, $W$ es el peso total del brazo inferior que actúa en el centro de gravedad del brazo inferior [17]. 


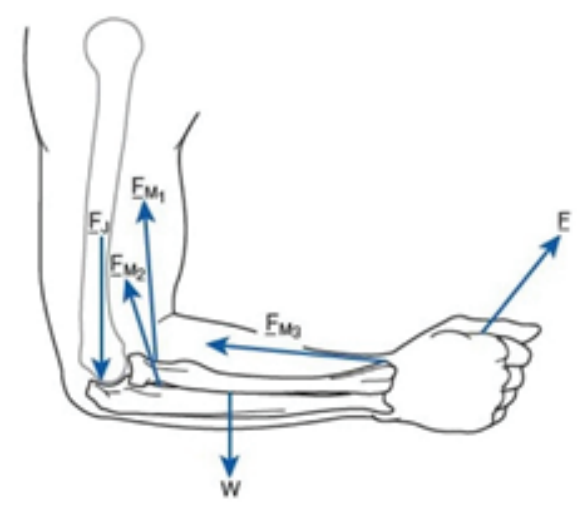

Figure 28

Diagrama de cuerpo libre de cargas sobre el brazo. Fuente: Basic biomechanics of the musculoskeletal system.

$F=>$ es la fuerza ejercida por los bíceps en el radio,

$F_{=?}$ es la fuerza ejercida por los músculos braquiorradiales en el radio,

$F_{=@}$ es la fuerza ejercida por los músculos braquiales sobre el cúbito, y

$F_{A}$ es la fuerza de reacción resultante en las articulaciones humeroulnar y humeroradial del codo.

Hay que tener en cuenta que las fuerzas de reacción muscular y articular representan los efectos mecánicos de la parte superior del brazo en la parte inferior del brazo. También tenga en cuenta que, como se ilustra en la Figura 28 (que no es un diagrama completo de cuerpo libre), las fuerzas de reacción de los músculos y articulaciones opuestas de igual magnitud, también actúan en la parte superior del brazo. Se considera el valor del peso igual a $50 \mathrm{~N}[18]$

\begin{tabular}{lccc}
\hline \multirow{2}{*}{ Músculo } & \multicolumn{3}{c}{ Parámetro } \\
\cline { 2 - 4 } & $F_{0 i}(\mathrm{~N})$ & $A_{i}(\mathrm{~cm} 2)$ & $S_{i}(\%)$ \\
\hline Braquial & 987,3 & 7,1 & $61 \%$ \\
Braquiorradial & 261,3 & 1,9 & $61 \%$ \\
Biceps largo & 624,3 & 4,5 & $61 \%$ \\
Bíceps corto & 435,6 & 3,1 & $61 \%$ \\
Triceps medio & 624,3 & 4,5 & $65 \%$ \\
Triceps lateral & 624,3 & 4,5 & $65 \%$ \\
Triceps largo & 798,5 & 5,7 & $65 \%$ \\
\hline
\end{tabular}

Figure 29

Propiedades de los músculos para los cálculos. Fuente: Basic biomechanics of the musculoskeletal system.

Acondicionamiento del modelo físico de acuerdo a las cargas actuantes y sujeciones [14]. 


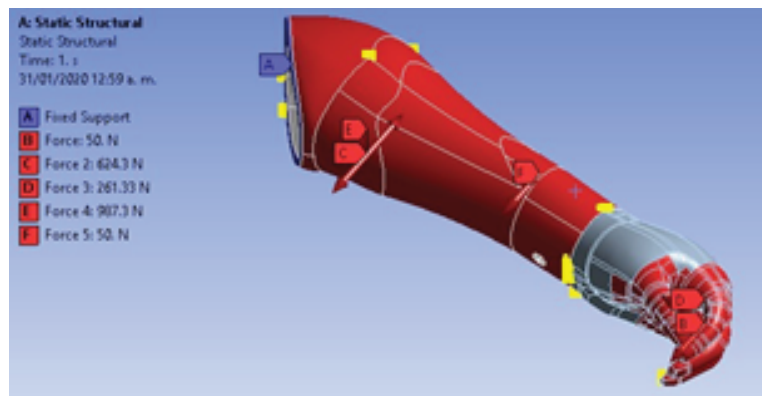

Figure 30

Prótesis y sus componentes para el análisis. Fuente: Autores.

\subsubsection{Proceso}

El computador genera el proceso de cálculo en base a las ecuaciones de energía de distorsión. En ANSYS MECHANICAL se muestra graficas en las que se puede analizar la convergencia de la solución. Uno de los factores importantes es el número 1743 de iteraciones que permitirá conducir a una mayor confiablidad 95\% de los resultados [15].

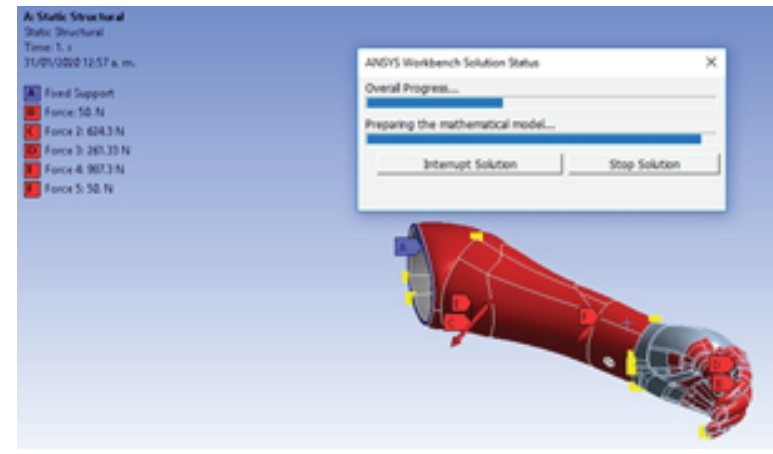

Figure 31

Resolución matemático. Fuente: Autores.

\subsubsection{Post proceso}

Ansys Mechanical genera como resultado de su simulación la combinación de esfuerzos mecánicos que se producen por la acción de las fuerzas antes mencionadas al igual que las deformaciones en las diferentes direcciones del modelo lo que permite observar el comportamiento de la prótesis cuando realice su función, permitiendo analizar el funcionamiento de la prótesis sin necesidad de la construcción.

La simulación permite obtener resultados del comportamiento estructural mecánico de la prótesis, en la Figura 32 se muestra el comportamiento de deformación cuando actúa sobre la prótesis las cargas descritas anteriormente, teniendo como resultado una deformación máxima de 1,2 mm. 


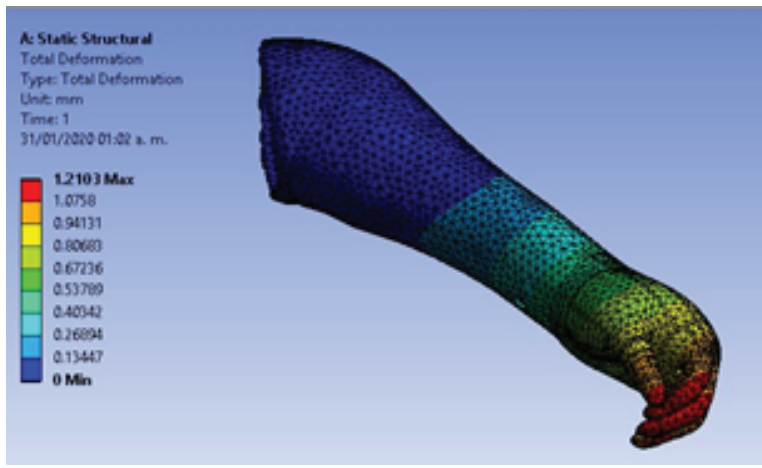

Figure 32

Deformación de la férula. Fuente: Autores.

Otro valor que se obtiene es el esfuerzo de von-mises que nos permite comprobar con el esfuerzo admisible del material utilizado, siendo un esfuerzo máximo de 4,13 Mpa, como se indica en la Figura 33.

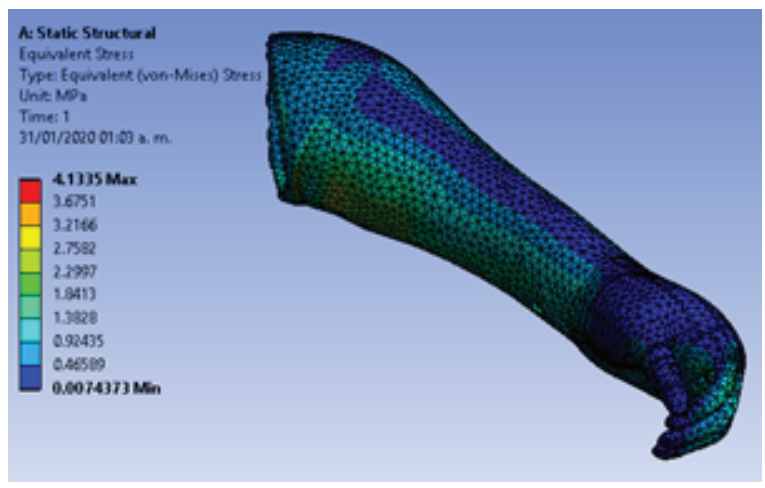

Figure 33

Esfuerzo de Von-Mises de la férula. Fuente: Autores.

Mantenemos un criterio de factor se seguridad para validar la resistencia teniendo como resultado un valor mayor a 15 , debido a que los esfuerzos generados por la acción de las fuerzas son menores al esfuerzo admisible de material que para el PLA es de $60 \mathrm{MPa}$, lo que indica que la prótesis se encuentra sobre diseñada para un análisis estático, como se muestra en la Figura 34 [19].

\section{Resultados y Discusión}

La utilización de un dispositivo para obtener la nube de puntos mediante el escaneo permite personalizar la prótesis.

Se consigue desarrollar el modelo 3D, gracias a una malla que no interfiere en la captura de la superficie principal de la geometría, debido a la flexibilidad para modelarse y mantener la forma. 


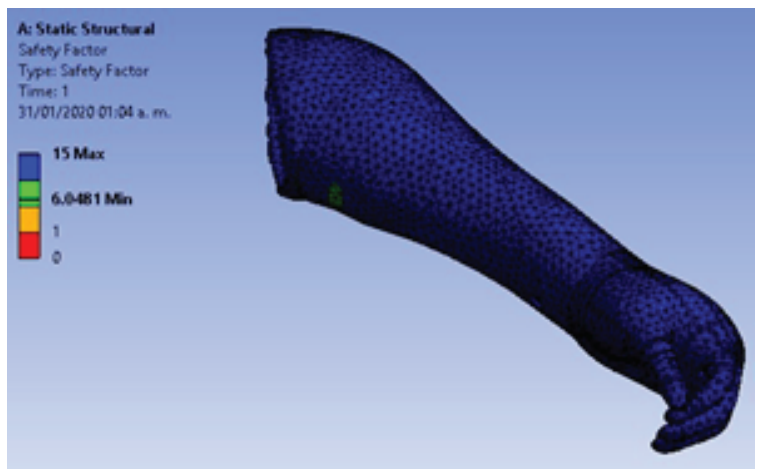

\section{Figure 34}

Factor de seguridad de la prótesis. Fuente: Autores.

La prótesis le ayudará en el desarrollo del volteo, apoyos, equilibrio, mejora de la fuerza de la musculatura proximal y a crear conciencia de la longitud del miembro, permitiendo sujetar objetos con las dos manos en la línea medía.

El sistema permite la colocación del muñón de una forma muy fácil y al mismo tiempo retirar el muñón con la misma condición.

\section{Conclusiones}

Se estableció las actividades para la obtención del modelo geométrico de la zona afectada muñón, mediante la aplicación de un scanner 3D, generándose de esta actividad un archivo STL el mismo que es considerado para la edición y creación de elementos sólidos para poder llevar a la fabricación y validación del diseño mediante el análisis computacional.

La creación del antebrazo se genera en un software CAD con herramientas de recubrir sólidos, para determinar su longitud se considera la simetría con el miembro existente (brazo).

La selección del material de impresión es el PLA debido a que es material no presenta contraindicaciones para el contacto con la piel según referencias de investigaciones.

La configuración del relleno para el proceso de impresión es importante para mantener la condición de masa en la prótesis y que proporcione la estabilidad de los miembros superiores, estableciéndose un valor de relleno de $30 \%$ y estructura interna de malla cubica para la impresión.

En la validación del diseño por simulación computacional, el comportamiento es aceptable porque se genera valores de deformación de 1,2 $\mathrm{mm}$ y un esfuerzo de 4,13 $\mathrm{MPa}$, considerando un factor de seguridad mayor a 15, esto se debe a que las propiedades mecánicas del PLA son las necesarias para generar prótesis de ese material. 
La prótesis construida mantiene un ajuste preciso en el momento de ser utilizada, debido a que se genera un vacío entre el socket y el muñón por las condiciones de personalización que esta prótesis.

\section{References}

[1] Periago RZ. Prótesis, ortesis y ayudas técnicas. Barcelona Editor Elsevier Masson. 2009;

[2] la Rosa Steinz R, Liptak L. Entrenador mioeléctrico de prótesis para amputados de brazo y mano. Mapfre Med. 2002;13(1):11-19.

[3] Brito JL, Quinde MX, Cuzco D, Calle Jl. Estudio del estado del arte de las prótesis de mano. 2013;

[4] Lucas LVM, Gennari FH, Goiato MC, Dos Santos DM, Moreno A, Falcón- Antenucci RM. Estética en prótesis removibles. Rev Cuba Estomatol. 2010;47(2):224-35.

[5] Loaiza JL, Arzola N. Evolución y tendencias en el desarrollo de prótesis de mano. Dyna. 2011;78(169):191-200.

[6] González JMD et al. Robótica y prótesis inteligentes. 2005;

[7] Osejos JVM. Caracterización de materiales termoplásticos de ABS y PLA semi-rgido impresos en 3D con cinco mallados internos diferentes. Quito; 2016.

[8] León M, Marcos-Fernández Á. Impresión 3D con materiales elástoméricos. Rev PLÁSTICOS Mod. 2019;118(747).

[9] Park C-S, Kim S-W, Kim D, Oh S-R. Comparison of plane extraction performance using laser scanner and Kinect. 8th International Conference on Ubiquitous Robots and Ambient Intelligence (URAI). 2011.

[10] Jaskulski A. Autodesk inventor professional 2016PL/2016/Fusion 360+ metodyka projektowania. Vol. 1. Wydawnictwo Naukowe PWN; 2015.

[11] Rodrigo SE, del Valle Herrera C. Una experiencia educativa en "ingeniería de rehabilitación" para generar competencias sobre ortoprotésica en ingeniería biomédica y bioingeniería.

[12] Talamantes Moreno EF. Diseño e Impresión 3D en Polimeros PLA y ABS de una prótesis mecánica con cierre voluntario a 2 tiempos para amputación de Mano. Licenciatura en Ing Biomédica. 2016;

[13] Ramirez NDM, Carbonell GRA, Ángel R et al. Simulación computacional como herramienta para disminuir los costos asociados al diseño mecánico. Retos la Dir. 2017;11(1):82-93.

[14] Alawadhi EM. Finite element simulations using ANSYS. CRC Press; 2015.

[15] Lee H-H. Finite element simulations with ANSYS workbench 18. SDC publications; 2018. 
[16] Nordin M, Frankel VH. Basic biomechanics of the musculoskeletal system. Lippincott Williams \& Wilkins; 2001.

[17] Latash ML. Neurophysiological basis of movement. Human Kinetics. 2008.

[18] Casás ZA. Estimación de fuerzas musculares durante el movimiento del brazo humano mediante optimización estático-fisiológica. 2016.

[19] Stolarski T, Nakasone Y, Yoshimoto S. Engineering analysis with ANSYS software. Butterworth-Heinemann; 2018. 